\title{
Research on Relationship between Chlamydia Trachomatis Infection and Female Infertility
}

\author{
Xiaojun Liu, Lili Xuan, Xiaofeng Wang
}

China-Japan Union Hospital of Jilin University, Jilin, Changchun, China, 130033

Keywords: Chlamydia Trachomatis Infection, Female Infertility, Relationship

\begin{abstract}
This paper investigates the relationship between genital C. trachomatis infection and female infertility. Chlamydia trachomatis and Ureaplasma urealyticum (UU) were detected by polymerase chain reaction (PCR) in 200 infertile patients and 100 normal births. Logistic regression analysis was performed. Results show that the positive rate of $\mathrm{C}$. trachomatis infection in infertility group (33.0\%) was significantly higher than that in the control group $(10.0 \%, \mathrm{P}<0.01)$. After treatment, the detection rate of $\mathrm{C}$. trachomatis negative pregnancy rate $(54.90 \%)$ was significantly higher than that of non-negative $(11.11 \%, \mathrm{P}<0.05)$. At the same time, the paper confirmed Chlamydia trachomatis infection and infertility, UU infection, cervical erosion $(\mathrm{P}<0.01)$. Reproductive tract Chlamydia trachomatis infection is one of the factors that cause infertility. Chlamydia trachomatis routine examination of infertile couples and timely treatment is necessary.
\end{abstract}

\section{Introduction}

Chlamydia trachomatis (CT) is a prokaryotic cell-borne microorganism that is parasitized in the genital tract and is a common pathogen of female genital tract infection [1]. In recent years, its infection rate has risen, which has become an important factor leading to women's infertility. To investigate the relationship between infertility and C. trachomatis infection in female genital tract, we used polymerase chain reaction (PCR) to detect Chlamydia trachomatis in 200 infertile patients and 100 healthy married women, Urea plasma urealyticum (urea plasma ureatylicum, UU) infection, cervical erosion and other related factors were analyzed in infertility group of Chlamydia trachomatis positive were observed and treated [1].

Reproductive tract infection is an important cause of infertility in women. Chlamydia trachomatis (CT) infection has drawn increasing attention from medical workers. Previous studies of infertility caused by CT infection were mostly serological or cervical PCR tests. In recent years, due to the rapid economic development, changes in lifestyle, people's life pressure increased significantly, the probability of female infertility increased significantly. UU and CT are the key pathogens causing female infertility during reproductive period. UU, CT are prokaryotes, in the female reproductive tract can cause inflammation of the genital organs and induce infection, leading to infertility. Due to UU and CT infection in women's genital tract after a longer duration and the disease prone to repeated, continuous development may lead to women's tubal mucosa and endometrial lesions occur, resulting in infertility.

CT is a very small bacteria specifically hosted in host cells that has a specific growth and reproduction cycle. Glandular epithelium of the genital tract such as endocervix and tubal endometrium are susceptible to CT infection. Ovovascular infection of CT, the tubal endometrial cell metabolism is inhibited, the cells were dissolved and necrosis, CT metabolites toxic to the cells and cause allergic reaction, the lipopolysaccharide on the CT membrane has an antigenic role in the host cell Evade immune rejection, and multiply quickly to cause persistent, stubborn infection that results in the following changes: ${ }^{1}$ Salpingitis: Edema, congestion, inflammatory exudation. Denied endometrial epithelial cell degeneration and necrosis; Chronic CT infection can cause severe tissue damage and subsequent scarring [2]. The pathological changes of the results, the tubal adhesions occurred in varying degrees, water, lumen obstruction, resulting in tubal ovulation, insemination, fertilized egg transport, nutrition, fertilized eggs and other loss of function and infertility. In this study, 23 cases of infertility patients with tubal CT infection, were found to have the above changes, 
on the other hand, tubal CT infection is often a chronic process. The vast majority of patients without obvious clinical symptoms and neglected, it should also be noted that when doing laparoscopy, who found tubal adhesions, tubal specimens should be taken for CT PCR detection. Hills reported that young is a risk factor for CT infection, especially those younger than 24 years of age, the main route of infection is abnormal sexual intercourse. This study found that female reproductive tract CT (cervix and fallopian tube) patients, 22 to 30-year-old group, accounting for $75 \%$, suggesting that young people are at high risk of CT infection. In addition, in terms of occupations, the study found that workers have the most CT sense, accounting for $52.3 \%$. Currently considered to prevent CT genital tract infection, mainly to prevent chaotic sexual intercourse, or use condoms during sex more secure. The study also suggested that CT infection and living area, duration of infertility, primary or secondary infertility and many other factors were not significantly related.

\section{Object and Method}

\subsection{The Object of Study}

June 2011 to June 2017 in our hospital there are gynecological clinic in 200 cases of infertility patients (infertility group). All exclude endocrine disorders, ovulation disorders and organic diseases, routine examination of their spouses normal. In the control group, 100 healthy women were given birth at the same period, aged from 23 to 39 years with a mean age of 31 years.

\subsection{The Method}

Specimen collection and treatment After fully exposed cervix, the first use of sterile cotton swabs outside the cervix secretions wipe clean, another take a special sterilized cotton swab inserted into the cervix outside the mouth, deep about $1 \sim 1.5 \mathrm{~cm}$, 360b rotation, stay for 30 seconds, remove and immediately insert the prepared sterile test tube. Then set $1 \mathrm{~mL}$ of saline to fully suspend, centrifuge at $1500 \mathrm{r}$ \# min-1 for $5 \mathrm{~min}$ and discard the supernatant. 5LL DNA extract was added into the pellet and mixed well, boiled for $10 \mathrm{~min}$, centrifuged at $1000 \mathrm{r}$ \# min-1 for $5 \mathrm{~min}$, and the supernatant 2LL was detected by PCR. 1.2.2 PCR detection PCR kit from Zhongshan Medical University Daan Gene Diagnostic Center. Take extracted DNA sample 2LL, $6000 \mathrm{r}$ min-1 centrifugation for a few seconds, at $93 \mathrm{e} 45 \mathrm{~s}, 55 \mathrm{e} 45 \mathrm{~s}, 72 \mathrm{e} 60 \mathrm{~s}, 35$ cycles, the final set 72e extended 5 min [3].

Determination of Results PCR amplified PCR tubes were taken from the PCR amplification product 10LL (no additional electrophoresis indicator), 2 \# L-1 agarose gel electrophoresis $20 \sim 30$ min, placed WP-92-1 UV analyzer observed, if the corresponding fluorescent bands appear (with the positive control at the same position) was positive.

Treatment of C. trachomatis test positive, given azithromycin $0.5 \mathrm{~g}$ intravenously once daily, the first doubled, once every $10 \mathrm{~d}$. Supplemented by vaginal spray net Kang rinse. If necessary, repeat a course of treatment. One week after discontinuation of CT review.

Statistical Analysis All experimental data input SPSS 8.0 software for statistical processing. Each group of data using V2 test for statistical analysis, the relevant variables using Logistic regression analysis, $\mathrm{A}=0.05$.

\section{The Relationship between Chlamydia Trachomatis Infection and Infertility}

Infertility is an important aspect of reproductive health research and its etiology is complex, many influencing factors. Female infertility associated with genital tract infections ranged from $20 \%$ to $60 \%$. Female genital tract of acute and chronic infections caused by genital tract inflammation, adhesions and obstruction is the main cause of infertility. Infection may be caused by a variety of microorganisms, of which Chlamydia trachomatis is one of the most common pathogens. It was found that $26 \%$ to $62 \%$ of acute salpingitis cases associated with Chlamydia trachomatis infection. In this study, the infection rate of Chlamydia trachomatis in infertility group was significantly higher than that of the control group. The pregnancy rate of Chlamydia trachomatis-negative patients after treatment was significantly higher than that of non-negative ones, 
suggesting that Chlamydia trachomatis infection is one of the important causes of female infertility. But Chlamydia trachomatis infection has nothing to do with the duration of infertility. Its pathogenic mechanism may be Chlamydia trachomatis infection inhibits the host cell metabolism, lysosomal release of cells was dissolved and destroyed, may also be related to the cytotoxic effects of metabolites caused by allergic reactions and autoimmunity [4].

Chlamydia trachomatis infection with UU infection aggravate infertility. Mycoplasma can not only cause widespread genital tract infections, leading to infertility, fetal infection, miscarriage and other adverse outcomes. And with UU infection, this study also confirmed this point, both exist at the same time may aggravate the degree of infertility. Hu Tao and others believe that Chlamydia trachomatis infection and UU infection on the activation of immune cells, although there is no cumulative cytokine production, but may be maintained for a long time. Chlamydia trachomatis, UU adhesion to sperm and its inflammatory damage to the genital tract, not only changed the fertilization environment, but also caused a local immune response. Some scholars believe that Chlamydia trachomatis, UU infection can induce T cells release of interferon, macrophage capture, sperm antigen presented immune response, leading to infertility.

According to incomplete statistics, in the world, $8 \%$ to $12 \%$ of infertile families account for $20 \%$ to $60 \%$ of infertility due to genital tract infections. The UU and CT are prokaryotic organisms, colonized in the genital tract, can cause genital tract infection, induced inflammatory response, which may eventually lead to infertility. Therefore, the study of UU and CT infection and female infertility has some clinical significance. UU has a certain adhesion, can adhere to the cell surface, release toxic substances to damage the host cells, to absorb the host cell nutrients supply their own growth and reproduction.

UU invades the mucous membrane of the female genital tract and adheres to the mucosal columnar epithelial cells. Once established, the UU will invade the mononuclear macrophages, destroy the cell structure and proliferate in the cell. After a large number of breeding, the infected person has an acute urinary tract infection to induce urethritis, cervicitis and other related diseases. Continued development of the disease, UU to upstream secondary infection of the uterus, fallopian tubes and pelvic cavity and other places, induced endometritis, salpingitis and pelvic inflammatory disease and other diseases, causing serious damage to women's reproductive health, may lead to female infertility. CT colonization in the reproductive tract, can inhibit the host cell metabolism, making the host cell metabolism disorder, the host cell lysosomal enzyme release, cell disintegration, resulting in toxic side effects of metabolites, but also may cause patients with their own super Sensitivity and immune response. In addition to infecting host cells, CT can infect neighboring cells and damage the cells by releasing toxic substances to adjacent cells, causing severe damage to the infected genital tract. UU, CT can damage sperm by releasing toxic substances and affect sperm motility by attaching to the surface of sperm. The possible mechanism is that the produced neuraminidase-like substance hinders sperm from binding to the egg [5].

Chlamydia trachomatis infection is an important factor in causing cervical erosion. This study shows that cervical erosion and Chlamydia trachomatis infection are closely related. The reason is that cervical columnar epithelium resistance is low, easy to invade the pathogen inflammation. Chlamydia trachomatis invade the body, the general first in the columnar epithelial growth and reproduction, damage to parasitic cells, leading to cervical erosion. However, $25 \%$ of women infected with $\mathrm{C}$. trachomatis do not show any symptoms and are easily overlooked. Among infertile couples worldwide, female infertility is associated with genital tract infections. UU can damage the cell membrane, affecting cell metabolism, invasion of the human body causes pelvic inflammatory disease, endometritis, annex inflammation caused by infertility. In addition UU can also affect the ability of sperm to penetrate the cervix, and damage the reproductive tract mucosa, so that immune cells contact with sperm, anti-sperm antibodies. In addition UU reproductive tract infections can stimulate female body produce anti-endometrial antibodies, a direct result of infertility. It is a special kind of pathogen, its pathogenic feature is easy to violate the columnar epithelial cells and along the line. Cause endometritis, salpingitis and tubal hydrops and other reproductive tract infections. Cervical tube is the most common site of infection, due to the hidden symptoms of 
infection, no more obvious clinical manifestations that lead to prolonged infection, resulting in genital tract injury. Therefore, the relationship between UU and female infertility has drawn more and more people's attention. The results showed that: UU, CT and UU CT positive rates in infertile women accounted for a very high rate, while the normal fertility of the control group of women, the positive rate was higher, the difference between the two groups was significant, Consistent with the current domestic reports, suggesting that UU, CT infection is one of the causes of female infertility, infertile women should routinely detect UU infection of the genital tract, positive patients should be based on drug sensitivity and timely treatment.

\section{Conclusions}

So for infertile women, especially those with cervical erosion of infertile women, screening and treatment of Chlamydia trachomatis is very necessary. Active prevention and treatment of Chlamydia trachomatis infection in women, to prevent the spread of sexually transmitted diseases, improve health is undoubtedly of great significance.

\section{References}

[1] Jiao Baoquan, Sun Yingli. Chlamydia trachomatis infection and female infertility [J]. Foreign Medical Sciences (Family Planning Volume). 2014 (03). 112.

[2] Zhang Jianping, Shao Rubin, Zhang Min, Zhu Aiping, Chang Xiufeng, Zhu Junjie. Studies on the Relationship between Infertility and Urea plasma urealyticum and Chlamydia trachomatis [J]. 2015 (10). 57-58.

[3] Yu Xinzhong, Wu Manwu, Yang Zhihao. 1, 500 cases of infertility infection in patients with Chlamydia trachomatis detection [J]. Journal of Infection Research. 2010 (09). 60-69.

[4] Li Lixia. Relationship between female infertility and Urea plasma urealyticum and Chlamydia trachomatis infections [J]. Tropical Medicine in China. 2016 (08). 135.

[5] Ruan Lixia. Chlamydia trachomatis infection and pregnancy [J]. China Hydropower Medicine. 2011 (01). 85-89. 